\title{
CJEM Debate Series: \#TPA should be the initial treatment in eligible patients presenting with an acute ischemic stroke
}

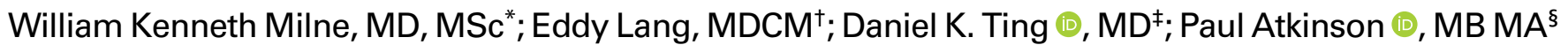

\section{INTRODUCTION}

\section{Daniel Ting (@tingdan) and Paul Atkinson (@eccucourse)}

This series of editorials provides CFEM readers with the opportunity to hear differing perspectives on topics pertinent to the practice of emergency medicine. The debaters have been allocated opposing arguments on topics where there is some controversy or perhaps scientific equipoise.

We continue with the topic of intravenous alteplase ( $\mathrm{tPA}$ - tissue plasminogen activator) in the treatment of acute ischemic stroke. Ischemic stroke is a leading cause of disability for Canadians, and the role of tPA in treatment has been one of the most hotly contested issues in our field over the past two decades. ${ }^{1,2}$ Although the rise of endovascular therapy (EVT) has shifted the conversation somewhat, tPA has broader indications and continues as a core component of treatment in stroke centres.

The Canadian Association of Emergency Physicians (CAEP) has recently changed its position on eligibility criteria for tPA in treating ischemic stroke. In 2015, CAEP gave a strong recommendation for the use of tPA within 3 hours of symptom onset and a weak/conditional recommendation against $\mathrm{PA}$ in the 3 - to 4.5 -hour time window. ${ }^{3}$ In 2018, the CAEP Board and Stroke Practice Committee endorsed the Level A Evidence recommendation for the use of tPA in patients presenting within 4.5 hours of symptom onset, provided by the Canadian Stroke Best Practices (CSBP) guideline group. ${ }^{4}$ The rationale for this change was that "the benefits of collaboration, including partnership in improved regional EVT pathways, outweigh the harms of [tPA] within the 3- to 4.5-hour treatment time window." "This change re-invigorated the debate of tPA in ischemic stroke on social media and across the country. This \#CFEMdebate article provides a longer-form medium, facilitating a more nuanced discussion.

Dr. Ken Milne is Chief of Staff of the South Huron Hospital Association, the creator of the Skeptics' Guide to Emergency Medicine website, and adjunct Professor at Western University. Dr. Milne argues that the evidence for the benefit of tPA has thus far been inadequate to reject the null hypothesis of no benefit, and, considering its high certainty of potential harm (bleeding), that emergency physicians ought to remain skeptical.

Dr. Eddy Lang is Professor and Department Head of the Calgary Zone and has chaired the last two CAEP position statements on stroke. He argues that not only is tPA the accepted, evidence-based treatment for ischemic stroke, but also that emergency physicians should be actively involved in the treatment and work collaboratively with stroke teams in developing stroke pathways.

Readers can follow the debate on Twitter and vote for either perspective, by going to @CFEMOnline or by searching \#CFEMdebate.

\section{INTRODUCTION}

"The evidence does not support a net patient-oriented benefit of thrombolysis for acute ischemic stroke."

\section{Ken Milne (@TheSGEM)}

To be clear, I am not "con" or against tPA for ischemic stroke but rather, skeptical. I do not make the claim that

From the *South Huron Hospital Association, Western University, London, ON; †Department of Emergency Medicine, University of Calgary, Calgary, AB; $¥$ Department of Emergency Medicine, University of British Columbia, Vancouver, BC; and the §Department of Emergency Medicine, Dalhousie University, Halifax, NS.

Correspondence to: Dr. Daniel K. Ting, Department of Emergency Medicine, University of British Columbia, Vancouver, BC V5Z 1M9, Canada; Email: daniel.ting@ubc.ca

(C) Canadian Association of Emergency Physicians

CJEM 2020;22(2):142-148

DOI 10.1017/cem.2020.3 
tPA does not work for stroke. Instead, I have not been convinced by the available evidence to reject the null hypothesis that thrombolysis has a net patient-oriented benefit in patients with stroke. The burden of proof is on those making the claim, and that burden has not been met. Therefore, I remain skeptical but willing to change my position if presented with more convincing evidence.

\section{Summary of the evidence}

The National Institute of Neurologic Disease Study (NINDS) is the study that could be credited with starting the $\mathrm{tPA}$ debate 24 years ago. ${ }^{6}$ This is the first and only randomized controlled trial (RCT) that reports a benefit of $\mathrm{tPA}$ for acute ischemic stroke under 3 hours. There were two parts to NINDS. Each part enrolled around 300 patients.

- NINDS, Part 1: The primary outcome was to see whether there was an improvement of 4 or more points on the National Institutes of Health Stroke Scale (NIHSS) score at 24 hours. They failed to demonstrate a statistical difference between $\mathrm{tPA}$ and placebo (i.e., it was a "negative trial").

- NINDS, Part 2: The primary outcome was a good neurologic outcome at 90 days. The results were $26 \%$ in the placebo group and $39 \%$ in the tPA group (an absolute difference of 13\%). There was also a $6 \%$ absolute increase in symptomatic intracranial hemorrhage (ICH).

The NINDS trials were limited by a relatively small sample size compared to the literature on thrombolysis in myocardial infarction, whose study populations were larger by one to two orders of magnitude. Furthermore, the groups were unbalanced on baseline stroke severity, which is a strong predictor of final stroke outcome. ${ }^{7}$

Hoffman and Schriger did a re-analysis of NINDS, Part 2, trying to control for some of the prognostic factors for outcome like stroke severity. The effect of tPA disappeared and the "time is brain" claim was not supported. ${ }^{8}$

The third European Cooperative Acute Stroke Study (ECASS-III) is the only other RCT of tPA for stroke that has reported benefit. ${ }^{9}$ They enrolled 821 patients who presented between 3 and 4.5 hours after symptom onset. ECASS-III showed a 7\% absolute benefit for a favorable outcome for efficacy. There was a $10 \%$ increase in any ICH with $\mathrm{tPA}$ and a $2 \%$ increase in symptomatic ICHs. Shy reported on the updated ECASS-III online results that report stroke history as a significant difference between the groups (14.1\% placebo v. $7.7 \% \mathrm{tPA}$ ), resulting in no benefit in the adjusted analysis. ${ }^{10}$ Also, if the endpoint is altered from the modified Rankin Scale (mRS) 0-1 versus 2-6, to a more realistic mRS 0-2 versus 3-6, the purported benefits of tPA disappear. $^{11}$

The third international stroke trial (IST-3) is the largest RCT in the tPA literature $(\mathrm{n}=3035) .{ }^{12}$ It was ethically approved for $\mathrm{tPA}$ in patients within 6 hours after symptom onset and included higher risk patients (over 80 years old). This was despite two other trials, ECASS-I and II, showing no benefit and increased harm (ICH and death). ${ }^{13,} 14$

IST-3 was a seriously flawed study for a number of reasons. ${ }^{15}$ The first 276 patients were double-blinded placebo controlled, and they found no statistical benefit. The study was then unblinded, which introduces bias. Patients were included if the clinician and patient agreed that the treatment was promising (selection bias).

The authors used a stroke severity score that is similar to the mRS. The reliability ${ }^{16}$ of the mRS is only moderate in trained clinicians, and its validity ${ }^{17}$ has been questioned. However, the outcomes for IST-3 were not done by trained clinicians but rather based on a mailed questionnaire or phone call to the unblinded patient. This lack of blinding introduces a placebo effect biasing the study towards efficacy. It is also unreasonable to expect the outcome assessment to be reliable and valid when unblinded stroke patients are self-reporting a subjective outcome.

The study was originally powered to have a sample size of 6,000 patients. Part way through the study, the authors realized 6,000 was no longer feasible. A second statistician persuaded the group that an ordinal method was both statistically more efficient and robust, rather than a dichotomous outcome (good v. bad neurologic outcome). Others argue that this is a flawed approach. ${ }^{18}$

The a priori primary dichotomous outcome of good neurologic function was under-powered and negative (no demonstrated statistical benefit). Adverse events and harms were increased with tPA. The number needed to harm (NNH) for bleeding was 17 and for an early death was 25 . Remarkably, the authors concluded that "despite the early hazards, thrombolysis within $6 \mathrm{~h}$ improved functional outcome. Benefit did not seem to be diminished in elderly patients." A more accurate conclusion is that "tPA within 6 hours failed to demonstrate improved functional outcome, and there was an increase in ICH and early mortality." 
More recently, the fragility index ${ }^{19}$ has been flagged in this debate. The fragility index of a study is the number of patients who would need to have a different outcome to cause the $p$-value to increase above 0.05 . The fragility index of the NINDS trial is 3 and ECASS-III is 1. A logical conclusion is that the bulk of published studies are correct - there is no clear benefit to tPA.

A summary of the thrombolysis literature is provided in Appendix A, recognizing that the evidence is more nuanced than a simple face emoji. However, there have been 12 studies with 1 positive $0-3$ hours, 1 positive 3-4.5 hours, 6 not demonstrating a clinically important benefit, and 4 stopped early due to harm or unlikely to prove beneficial.

In the hierarchy of evidence-based medicine, systematic reviews and meta-analyses are put above RCTs. ${ }^{20} \mathrm{I}$ do not agree completely with this hierarchy because systematic reviews and meta-analyses depend on a number of things, such as the quality of included and excluded studies. Many of the evidence syntheses of the thrombolysis literature do not seem to get us any closer to the "truth" about tPA for stroke. ${ }^{21}$

However, one systematic review and meta-analysis by Wardlaw et al. found no statistical difference between thrombolytic agents for stroke. ${ }^{22}$ This finding is important because the systematic review and meta-analysis by Emberson et al. excluded any studies not using tPA as the thrombolytic agent. ${ }^{23}$ Furthermore, Emberson et al. included the seriously flawed IST $-3 .^{23}$ It is hard to understand how the negative IST-3 study does not move the totality of the evidence in an even more negative direction.

Emberson et al. also reported multiple conflicts of interest. This does not negate their interpretation, but industry-supported studies are associated with more favorable results and conclusions than sponsorship by other sources. $^{24,25}$ The Institute of Medicine published guidelines on systematic reviews and meta-analyses and recommends the exclusion of "individuals with a clear financial conflict of interest." 26

Donaldson et al. completed a systematic review and meta-analysis looking at the thrombolytic literature, reported no conflicts of interest, came to a skeptical conclusion, and encouraged more RCTs. ${ }^{27}$ My interpretation of the literature is closer to Donaldson et al.'s than Emberson et al.'s.

\section{Time is brain}

There is an association between time and the brain, but a causal relationship has not been established. Perhaps those treated earlier are more likely to have a transient ischemic attack and therefore do better. Even the seriously flawed IST-3 subgroup analysis did not support the claim of time is brain (Appendix B). A salvageable brain on advanced neuroimaging is brain. It does not matter how much time has passed since the symptom onset if there is no viable brain to re-perfuse.

\section{Guidelines}

Guidelines should guide not dictate our care. The American College of Emergency Physicians (ACEP) recently revised their guidelines due to concerns from their members. ${ }^{28}$ They improved the management of conflicts of interest, added methodologists, and improved adherence to rating methodology. The older ACEP guidelines gave a Level A recommendation for the use of $\mathrm{tPA}$ under 3 hours and a Level $\mathrm{B}$ if given between 3 and 4.5 hours. The new ACEP guidelines downgraded the recommendations to Level $\mathrm{B}$ for patients presenting under 3 hours. ${ }^{29}$

The CSBP guidelines were published in International Fournal of Stroke in 2018. ${ }^{4}$ However, International Fournal of Stroke did not seem to adhere to the Institute of Medicine guidelines on managing conflicts of interest in the creation of the guideline. ${ }^{26}$ Specifically, it appears that the co-chairs of the guideline working group disclosed multiple conflicts of interest with the pharmaceutical industry. This included Boehringer Ingelheim that markets and sells tPA. ${ }^{4,30}$

CFEM published a synopsis of the CSBP guidelines in 2018. ${ }^{5}$ The authors endorse a Level A recommendation for tPA in eligible patients with symptom onset under 4.5 hours. No competing interests were reported in this publication. However, three of the authors were on both the CSBP guideline paper and the CFEM synopsis paper. These individuals reported conflicts of interest with the pharmaceutical industry and specifically with Boehringer Ingelheim on the CSBP guidelines publications. ${ }^{4,30}$ It is unclear as to why these conflicts of interest were not reported in the $C F E M$ synopsis publication.

\section{CONCLUSION}

To restate my position, I do not make the claim that $\mathrm{tPA}$ does not work for patients with stroke. That would be a logical fallacy by shifting the burden of proof. Science starts with the null hypothesis (no effect). Advocates for tPA have the burden and have not reached it. 
It is hard to get an established medical practice changed. Unfortunately, tPA advocates have significantly changed emergent stroke care based on weak evidence. Objectively looking at the available literature suggests equipoise, at best. We urgently need replication of NINDS/ECASS-III to either confirm or refute the findings. Until such time, I would suggest that CAEP review their current guidelines, remove those individuals with conflicts of interest, include methodologists, improve the adherence to rating methodology, and update the CAEP recommendations to reflect the strength of the available evidence. $^{31}$

\section{INTRODUCTION}

"Thrombolysis has a role in stroke but outcomes hinge on emergency physician involvement."

\section{Eddy Lang (@EddyLang1)}

Arguably the most divisive debate in our speciality, the argument about thrombolysis for acute ischemic stroke has raged at times but has persisted for over two decades. Before considering a synopsis of the evidence, it is worth asking: What has the debate achieved? Has the debate contributed to improvements in patient care? Has it enhanced the place of emergency medicine in the house of medicine? The answers will be a matter of perspective, of course, but I would argue that the downsides of the debate have exceeded the benefits, and it may be time to put the divisiveness that the debate has yielded behind us.

\section{Summary of the evidence}

The tPA benefits are highly time-dependant, hence the importance of considering the evidence as a function of the door to needle time. For tPA within 3 hours of last seen normal, we have individual patient-level RCT data on 1,549 patients enrolled from seven RCTs demonstrating a clear time-dependent effect. The same is suggested from real-life observational data from registries for many thousands more showing better outcomes with high rates of $\mathrm{tPA}$ use. ${ }^{23,32}$ For $\mathrm{tPA}$ given from 3 - to 4.5-hours, there are 2,768 patients enrolled in 11 trials that contribute to our understanding of efficacy, as well as extensive real-world data. ${ }^{33}$ Counting the contributing studies with varying inclusion criteria as scoring positive or negative, like wins and losses for a hockey team, is inconsistent with systematic review methodology.

The evidence has been deemed of moderate to high certainty for treatment within 3 hours by guideline panels, as well as by critics of tPA in stroke. In other words, a consistent effect size around benefits and harms with reasonable precision is well established; it is not bulletproof but a robust dataset in comparison to many of the things that we treat in the emergency department (ED). ${ }^{27}$

The likelihood of achieving an excellent outcome (mRS 0-1) after acute stroke is increased by $9.8 \%$, or a number needed to treat (NNT) for benefit of 10 if treated within 3 hours. The benefit comes with a risk of harm ranging from $2 \%$ to $2.5 \%$ or an NNH of 40 to 50 .

The certainty in evidence is considerably less for treatment between 3 and 4.5 hours. The benefit is unquestionably diminished in the delayed time range, whereas bleeding risk is essentially unchanged. The NNT for benefit is 20 and the NNH is 40 to $50 .^{33}$ This narrow balance between benefit and harm at 3- to 4.5-hours is why the CAEP Stroke Practice Committee recommended against lysis beyond that time window in our 2015 guideline. ${ }^{3}$

\section{Thrombolysis is a risky treatment that is sensitive to patient preferences}

Administering a treatment that carries significant risks to achieve a longer-range gain is not in the emergency medicine physician wheelhouse. Cardiac surgeons and oncologists using cytotoxic regimens expose patients to significant short-term risks on a regular basis to achieve a long-term benefit, but we do not. It is understandably difficult for us to accept that there may be patients who would accept the risk of harm to achieve a more likely chance of benefit.

Given the risks of tPA, it is imperative that patients and family (often the witnesses to last seen normal) are consulted and undergo an informed consent prior to thrombolysis administration. Patients who suffer ischemic stroke or who are consulted based on a theoretical scenario will generally accept the risks of fatal or symptomatic ICH, to obtain the potential benefits of functional independence and an excellent outcome. ${ }^{34}$ Ask your older loved ones what they would want.

However, consent cannot be assumed, and tPA should never be administered without understanding the likelihood of benefit and the chances of being harmed. 
Admittedly, we lack precise tools to convey this information to patients, or more likely their families, in the event that they are incapacitated and unable to weigh in on the decision, and families need to know that the likelihood of benefit is significantly less if treated beyond the 3-hour window, and that the treatment can make them worse. Patients who value functional independence above other outcomes will accept this treatment.

\section{IST-3 and its problematic contribution}

Critics of tPA in stroke have highlighted a number of issues. A common issue relates to the IST-3 trial, which poses both an increased risk of bias through its outcome reporting and demonstrated a paradoxical worsening in functional outcome in placebo-treated patients in the 3- to 4.5-hour time window. IST-3 and its inconsistent results at 3 to 4.5 hours are the primary contributor to the uncertainty in evidence for treatment in this time window. Yet it meets eligibility criteria for all systematic review and guideline projects on the topic and, if excluded from the evidence base, would only strengthen the effect for treatment at 3 to 4.5 hours. ${ }^{12}$

\section{Where does the burden of proof lie?}

A common argument of tPA detractors is that the burden of proof lies with those claiming benefit. In 2019, tPA is used to treat acute ischemic stroke in nearly every jurisdiction with the capability of doing so, and all government-sponsored clinical practice guidelines endorse its use. If there is to be a change in practice, it should be up to those opposed to the treatment to raise sufficient doubts to have the current state reconsidered. It would be a truly impressive and remarkable achievement if those who carry the skepticism mantra towards tPA were to trigger a Health Canada review of the product or convince a funding body to support further research into the benefits of tPA in comparison to no treatment. Unfortunately, this has not come about.

\section{What should the role of the emergency medicine physician be in acute stroke?}

Emergency medicine physicians play an indispensable role in this exquisitely time-sensitive condition. For one, they can contribute to the efficient ascertainment of eligibility and informed consent working in tandem with their stroke neurologist colleagues. Two minds, informed of the nuances of stroke treatment with thrombolytics, including benefits and risks, will always supersede a single perspective, and a collaborative approach is always preferred. As leaders of the ED, we are in a unique position to ensure that door to needle times are safely optimized in eligible patients.

As we now enter the EVT era, it is particularly important that patients know about all options available to them. It is up to the entire treating team to determine the specific risk profile of a given patient so that the decision to proceed with tPA and/or EVT is optimized to meet patient preferences when large vessel occlusion is present. ${ }^{1,35}$

\section{What does the future of acute stroke care look like?}

The treatment that we offer patients is invariably based on the best available evidence that we have at the time. In 2019, there was equipoise around the question of whether tPA adds benefit as a bridging therapy in EVT, and the trials examining this are ongoing.

Until this is clarified, the core argument that we are presenting is that patient care is optimized when ED and Stroke teams collaborate and share a common strategy and interpretation of the evidence. If you remain skeptical and thus uncertain about being pro or con and, despite engagement, remain unable to convince your colleagues in stroke care that the burden of proof has not been met, it may be in the best interests of the patient and the whole team to leave any misgivings at the door of the ED.

A jointly developed Code Stroke protocol is the cornerstone of good patient care in 2019. Achieving this requires an acceptance that $\mathrm{tPA}$ can be considered a standard of care in consenting and eligible patients and families. Standing on the sidelines while our Stroke colleagues unilaterally make all of the critical decisions for these patients is not acceptable. Failure to accept tPA as a standard of care will only further marginalize our role in the emergency care of patients with stroke.

Healthy skepticism as opposed to its unhealthy counterpart is an integral part of critical thinking. The state of evidence as it exists in 2019 is that tPA delivered in a timely manner improves outcomes from acute ischemic stroke, but only in patients or surrogates who understand and agree to the risks involved.

Acknowledgements: The authors acknowledge Devin Harris for his critical reading of the manuscript.

Keywords: Debate, emergency medicine, stroke, thrombolysis 
Competing interests: Dr. Milne serves as a senior editor of $A c a-$ demic Emergency Medicine. He has no funding from the pharmaceutical or biomedical device industry. $\mathrm{He}$ is on the faculty for the Center for Medical Education and EM:RAP. Dr. Milne does partake in medical malpractice reviews and does hold a patent on a pediatric resuscitation device. Dr. Lang reports a potential intellectual conflict of interest in regard to his role as Chair of the CAEP Stroke Practice Committee since 2014. Dr. Lang also reports educational activities sponsored by the industry and that, since 2012, all funding associated with this has been directed to both the CAEP Research Fund and the Emergency Medicine Research Fund within the Calgary Health Trust. This includes funds received from CAEP accredited symposia sponsored by Boehringer Ingelheim (makers of alteplase) but related to a bleeding reversal agent and not ischemic stroke.

\section{REFERENCES}

1. Harris DR, Lang ES, Perry JJ, Morrison LJ. Treatment of stroke in Canadian emergency departments: time to be leaders. CFEM 2017;19(1):47-9.

2. Johnstone C. Thrombolysis for acute ischemic stroke: does it work? - the con position. CFEM 2015;17(2):180-3.

3. Harris D, Hall C, Lobay K, et al. Canadian Association of Emergency Physicians position statement on acute ischemic stroke. CFEM 2015;17(2):217-26.

4. Boulanger JM, Lindsay MP, Gubitz G, et al. Canadian stroke best practice recommendations for acute stroke management: prehospital, emergency department, and acute inpatient stroke care, 6th edition, update 2018. Int 7 Stroke 2018;13 (9):949-84.

5. Lin K, Lindsay P, Shams T, et al. A summary of the Canadian stroke best practice recommendations, sixth edition (2018): updates relevant to prehospital and emergency medicine providers. CFEM 2018;20(5):685-92.

6. The National Institute of Neurological Disorders and Stroke rt-PA Stroke Study Group. Tissue plasminogen activator for acute ischemic stroke. N Engl 7 Med 1995;333(24):1581-7.

7. Adams Jr HP, Davis PH, Leira EC, et al. Baseline NIH Stroke Scale score strongly predicts outcome after stroke: a report of the Trial of Org 10172 in Acute Stroke Treatment (TOAST). Neurology 1999;53(1):126-31.

8. Hoffman JR, Schriger DL. A graphic reanalysis of the NINDS trial. Ann Emerg Med 2009;54(3):329-36, 36 e1-35.

9. Hacke W, Kaste M, Bluhmki E, et al. Thrombolysis with alteplase 3 to 4.5 hours after acute ischemic stroke. $N$ Engl 7 Med 2008;359(13):1317-29.

10. Shy BD. Implications of ECASS III error on emergency department treatment of ischemic stroke. 7 Emerg Med 2014;46(3):385-6.

11. Ferguson C. Use of thrombolysis in acute ischemic stroke; updated April 14, 2010. Available at: https://bestbets.org/ bets/bet.php?id=717\&hash (accessed September 24, 2019).

12. The IST-3 Collaborative Group. The benefits and harms of intravenous thrombolysis with recombinant tissue plasminogen activator within $6 \mathrm{~h}$ of acute ischaemic stroke (the third international stroke trial [IST-3]): a randomised controlled trial. Lancet 2012;379(9834):2352-63.
13. Hacke $W$, Kaste $M$, Fieschi C, et al. Intravenous thrombolysis with recombinant tissue plasminogen activator for acute hemispheric stroke. The European Cooperative Acute Stroke Study (ECASS). 7AMA 1995;274(13):1017-25.

14. Hacke $W$, Kaste $M$, Fieschi C, et al. Randomised doubleblind placebo-controlled trial of thrombolytic therapy with intravenous alteplase in acute ischaemic stroke (ECASS II). Second European-Australasian Acute Stroke Study Investigators. Lancet 1998;352(9136):1245-51.

15. Hoffman JR, Cooper RJ. How is more negative evidence being used to support claims of benefit: the curious case of the third international stroke trial (IST-3). Emerg Med Australas 2012;24(5):473-6.

16. Wilson JT, Hareendran A, Hendry A, et al. Reliability of the modified Rankin Scale across multiple raters: benefits of a structured interview. Stroke 2005;36(4):777-81.

17. Zhao H, Collier JM, Quah DM, Purvis T, Bernhardt J. The modified Rankin Scale in acute stroke has good inter-rater-reliability but questionable validity. Cerebrovasc Dis 2010;29(2):188-93.

18. Radecki RP, Chathampally YG, Press GM. rt-PA and stroke: does IST-3 make it all clear or muddy the waters? Answers to the November 2012 Journal Club questions. Ann Emerg Med 2013;61(4):489-98.

19. Walter SD. Statistical significance and fragility criteria for assessing a difference of two proportions. 7 Clin Epidemiol 1991;44(12):1373-8.

20. Murad MH, Asi N, Alsawas M, Alahdab F. New evidence pyramid. Evid Based Med 2016;21(4):125-7.

21. Fatovich DM. Truth, thinking and thrombolysis. Emerg Med Australas 2016;28(5):490-2.

22. Wardlaw JM, Koumellis P, Liu M. Thrombolysis (different doses, routes of administration and agents) for acute ischaemic stroke. Cochrane Database Syst Rev 2013(5):CD000514.

23. Emberson J, Lees KR, Lyden P, et al. Effect of treatment delay, age, and stroke severity on the effects of intravenous thrombolysis with alteplase for acute ischaemic stroke: a meta-analysis of individual patient data from randomised trials. Lancet 2014;384(9958):1929-35.

24. Lundh A, Sismondo S, Lexchin J, Busuioc OA, Bero L. Industry sponsorship and research outcome. Cochrane Database Syst Rev 2012;12:MR000033.

25. Hansen C, Lundh A, Rasmussen K, Hrobjartsson A. Financial conflicts of interest in systematic reviews: associations with results, conclusions, and methodological quality. Cochrane Database Syst Rev 2019;8:MR000047.

26. Graham R, Mancher M, Wolman DM, Greenfield S, Steinberg E, eds. Clinical practice guidelines we can trust. Washington, DC: National Academies Press; 2011.

27. Donaldson L, Fitzgerald E, Flower O, Delaney A. Review article: why is there still a debate regarding the safety and efficacy of intravenous thrombolysis in the management of presumed acute ischaemic stroke? A systematic review and meta-analysis. Emerg Med Australas 2016;28(5):496-510.

28. Radecki RP. ACEP Clinical policy on intravenous tissue plasminogen for stroke continues to evolve. ACEP Now; 2015. Available at: https://www.acepnow.com/article/acep-clinical-policy-on-intravenous-tissue-plasmogen-for-strokecontinues-to-evolve/ (accessed September 4, 2019). 
29. American College of Emergency Physicians, American Academy of Neurology. Clinical policy: use of intravenous tPA for the management of acute ischemic stroke in the emergency department. Ann Emerg Med 2013;61(2):225-43.

30. Casaubon LK, Boulanger JM, Blacquiere D, et al. Canadian stroke best practice recommendations: hyperacute stroke care guidelines, update 2015. Int $\mathcal{f}$ Stroke 2015;10 (6):924-40.

31. Lenzer J, Hoffman JR, Furberg CD, Ioannidis JP. Guideline Panel Review Working Group. Ensuring the integrity of clinical practice guidelines: a tool for protecting patients. BM7 2013;347:f5535.

32. Qin B, Zhao MJ, Chen H, et al. Real-world outcomes of acute ischemic stroke treatment with intravenous thromboly- sis: a systematic review and meta-analysis. 7 Stroke Cerebrovasc Dis 2018;27(12):3542-8.

33. The NNT.com. Available at: https://www.thennt.com/nnt/ thrombolytics-acute-ischemic-stroke/ (accessed September 4, 2019).

34. Flatharta TO, Khan A, Walsh T, O'Donnell M, O'Keefe ST. Advance preferences regarding thrombolysis in patients at risk for stroke: a cross-sectional study. Q7M 2015;108 (1):27-31.

35. Kamal N, Holodinsky JK, Stephenson C, et al. Improving door-to-needle times for acute ischemic stroke: effect of rapid patient registration, moving directly to computed tomography, and giving alteplase at the computed tomography scanner. Circ Cardiovasc Qual Outcomes 2017;10(1):e003242.

Appendix A Summary of the literature of thrombolytics for treatment of acute ischemic stroke

\begin{tabular}{|c|c|c|c|c|c|}
\hline Trial name $(n)$ & Journal (year) & Time (h) & Benefit & Harm & Emoji \\
\hline $\begin{array}{l}\text { MAST } \\
\qquad(n=622)\end{array}$ & Lancet (1995) & $<6$ & None & Increased early death & ;) \\
\hline $\begin{array}{l}\text { ECASS-I } \\
\qquad(n=620)\end{array}$ & JAMA (1995) & $<6$ & None & Benefit does not outweigh risk & :) \\
\hline $\begin{array}{l}\text { NINDS-1 } \\
\qquad(n=291)\end{array}$ & NEJM (1995) & $<3$ & None & No difference & ;) \\
\hline $\begin{array}{l}\text { NINDS-2 } \\
\qquad(n=333)\end{array}$ & NEJM (1995) & $<3$ & $\sim 13 \%$ benefit at 90 days & Increased ICH & (2) \\
\hline $\begin{array}{l}\text { MAST-Eu } \\
\qquad(\mathrm{n}=310)\end{array}$ & NEJM (1996) & $<6$ & None & Stopped early due to harm & (2) \\
\hline $\begin{array}{l}\text { ASK } \\
\qquad(n=340)\end{array}$ & JAMA (1996) & $<4$ & None & Stopped early due to harm & (2) \\
\hline $\begin{array}{l}\text { ECASS-II } \\
\qquad(\mathrm{n}=800)\end{array}$ & Lancet (1998) & $<6$ & None & No difference & :) \\
\hline ATLANTIS-B $(n=613)$ & JAMA (1999) & $3-5$ & None & Stopped early & (:) \\
\hline ATLANTIS-A $(n=412)$ & Stroke (2000) & $<6$ & None & Stopped early due to harm & (:) \\
\hline $\begin{array}{l}\text { ECASS-III } \\
\qquad(\mathrm{n}=621)\end{array}$ & NEJM (2008) & $3-4.5$ & $7 \%$ benefit at 90 days & Increased ICH & (-) \\
\hline $\begin{array}{l}\text { DAIS-2 } \\
\qquad(n=193)\end{array}$ & Lancet (2009) & $3-9$ & None & No difference & $:)$ \\
\hline $\begin{array}{l}\text { IST-3 } \\
\qquad(\mathrm{n}=3035)\end{array}$ & Lancet (2012) & $<6$ & None & No difference & :) \\
\hline
\end{tabular}

Appendix B Adjusted effect of treatment on the primary outcome (alive and independent, Oxford Handicap Score 0 , 1, or 2) in $\underline{\text { subgroups }^{12}}$

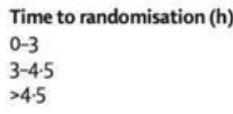

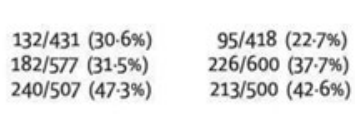

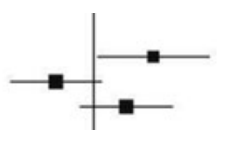

$1.64(1.03-2.62)$
$0.73(0.50-1.07)$
$1.31(0.89-1.93)$
0.613 